\title{
Ante-proyecto de Ley Marco para las empresas recuperadas por los trabajadores
}

\author{
Alberto García Müller \\ amuller@ula.ve \\ Universidad de los Andes. Venezuela
}

Recibido: 26.06 .09

Aceptado: 7.07 .09

Resumen: El presente trabajo constituye un anteproyecto de normativa de regulación especializada de las empresas recuperadas por los trabajadores, para que sea sometida a discusión y aprobación como Ley Marco por el Parlamento Latinoamericano, de manera que los Estados miembros se comprometan a sancionar en sus respectivos órganos legislativos leyes sobre el particular.

Palabras clave: ley, marco, empresa, recuperada, trabajadores.

Abstract: The present work constitutes a first draft of norm of regulation specialized of the companies recovered by the workers, so that it is put under discussion and approval like Law Marco by the Latin American Parliament, so that the States members are committed to sanction in their respective legislative organs laws on the individual.

Key words: law, frame, company, recovered, working. 
Este anteproyecto fue elaborado por el autor por cuenta del Grupo Parlamentario Venezolano del Parlamento Latinoamericano, una vez analizadas las observaciones de los miembros de la comisión ad-hoc designada por el mismo, en especial, de la Dra. Neyda Delgado, los aportes formulados por el Ministerio del Trabajo de Venezuela y las conclusiones del Primer Encuentro Latinoamericano de Empresas Recuperadas, celebrado en Caracas, todo ello en el mes de octubre de 2005.

La presente versión actualiza sus contenidos de acuerdo a la revisión de la bibliografía, las opiniones de expertos y de directivos de empresas recuperadas, así como de acuerdos adoptados en diversos encuentros de trabajadores de las mismas, fundamentalmente de la Argentina, y trata de reflejar sus planteamientos, necesidades y requerimientos según los criterios personales del autor.

Cabe destacar que - de acuerdo a nuestro conocimiento- después del encuentro de Caracas no se han producido hechos que demuestren la voluntad de aprobar una Ley Marco de Empresas Recuperadas para el área latinoamericana por los responsables de hacerlo, lo cual nos hace pensar en el carácter meramente político de la iniciativa. Lo que es lamentable, dada la necesidad y urgencia de dotar a esta forma jurídica de un marco regulatorio adecuado a su desarrollo y consolidación, y que tantos beneficios ha traído a los trabajadores.

\section{CAPÍTULO PRIMERO \\ Disposiciones Generales}

\section{Artículo 1. Objeto de la Ley}

La presente Ley tiene por objeto establecer un marco regulatorio de referencia que permita a los Estados, en orden a la activación del aparato productivo nacional, la democratización de las relaciones de producción y el fomento de nuevas formas productivas, dictar normativas sobre las empresas recuperadas por los trabajadores con la finalidad de:

a) Dotarlas de un marco legal adecuado para fortalecer su promoción, consolidación y desarrollo autónomo. De igual manera, regular la ocupación por parte de los trabajadores de empresas al momento del cierre, para asegurar el pago de los créditos laborales pendientes, la preservación de los activos empresariales y la cancelación de las obligaciones fiscales y parafiscales insolutas; 
b) Contribuir al mantenimiento y creación —en forma sostenidade puestos de trabajo decente a través de la preservación e incremento de estructuras productivas y de servicios recuperadas por los trabajadores que se encuentren en funcionamiento o en proceso de reactivación;

c) Evitar que por una deficiente regulación sea posible la auto o la hetero explotación de los trabajadores a través de seudo empresas asociativas utilizadas como mecanismos de simulación para cometer fraude laboral;

d) Crear sistemas y procedimientos que garanticen un coherente y efectivo apoyo del Estado nacional, regional y local a estas empresas;

e) Incrementar nuevas relaciones sociales y de producción auto gestionadas, para ampliar demandas, necesidades y experiencias comunes.

\section{Artículo 2. La Empresa Recuperada}

a) A los efectos de la presente Ley, son empresas recuperadas por los trabajadores aquellas empresas en cesación de actividades, vaciamiento por parte de los patronos o de cierre por cualquier causa, que se encuentran en proceso de rehabilitación por parte de sus trabajadores como medida para preservar sus puestos de trabajo, evitar el cese de sus actividades productivas o reiniciar las mismas;

b) Las empresas recuperadas se constituirán legalmente bajo la forma jurídica que consideren más adecuada a su situación concreta, preferentemente cooperativas de trabajo asociado;

c) Deberán ser auto gestionadas por los trabajadores, aunque podrán participar en procesos de cogestión con empresas privadas y, o con entidades del sector público, en diversos grados y condiciones, así como celebrar convenios de concentración o de colaboración empresarial con los mismos;

d) Se declara de interés o utilidad pública, social o nacional el proceso de recuperación de empresas por parte de los trabajadores;

e) Los créditos laborales de los trabajadores tienen el carácter de alimentarios y gozarán de privilegio de primer grado.

\section{Artículo 3. Características}

Las empresas recuperadas se caracterizan por: 
a) Sustentar su actuación en los valores de la solidaridad, la cooperación, la participación, la equidad, la corresponsabilidad y la protección del ambiente;

b) La libertad de ingreso y de retiro de los trabajadores miembros, sujeta a la capacidad productiva y, o de servicios de la empresa;

c) La adaptación de procedimientos internos democráticos en la toma de decisiones;

d) La equidad en las aportaciones económicas de los trabajadores, en sus remuneraciones y en el reparto de utilidades o excedentes obtenidos, todas las que constituyen el límite de la responsabilidad patrimonial de los miembros por las obligaciones sociales;

e) La provisión de servicios de protección social a sus miembros y a sus grupos familiares;

f) Gozan de autonomía organizativa y de independencia en el ejercicio de sus actividades;

g) Efectúan todo tipo de actividad económica, social o cultural lícita, en igualdad de condiciones con las demás empresas de la forma jurídica que fuesen;

h) Proveen servicios y promueven actividades socioeconómicas dirigidas a mejorar su entorno y calidad de vida de las comunidades, para lo cual invierten parte de sus excedentes;

i) Colaboran con otras empresas recuperadas y con otras formas de participación social, así como con la comunidad en donde actúan;

j) Así mismo, proveerán la equidad de género en las responsabilidades directivas, ejecutivas y operativas.

\section{Artículo 4. Fuentes}

a) Las empresas recuperadas se regirán de acuerdo al siguiente orden normativo: la presente Ley en la materia de la especialidad; en lo sustantivo, por las normas legales de la forma jurídica seleccionada, la legislación en materia de trabajo y seguridad social que fuere procedente, y por sus propios estatutos y reglamentos internos, en especial, el reglamento de trabajo;

b) Los litigios que surjan con motivo de la aplicación de esta Ley serán del conocimiento de la autoridad judicial competente de acuerdo a la forma jurídica seleccionada, y se seguirán por el procedimiento establecido en dichas Leyes. 


\section{Artículo 5. Causales}

\section{CAPÍTULO SEGUNDO \\ Procedencia}

Se considera una empresa susceptible a ser recuperada por los trabajadores cuando se encuentre en situación de pérdida efectiva, o de grave e inminente peligro de pérdida de los puestos de trabajo, o esté atravesando problemas que hagan comprometer su propia existencia y sea imprescindible hacerlo, cuando concurran algunas de las siguientes causales:

a) El cierre unilateral de la empresa o lock out total o parcial de sus actividades u operaciones, o el despido masivo de trabajadores, sin causa justificada;

b) La obsolescencia evidente de los equipos, maquinarias y, o de los procedimientos técnico-productivos utilizados, o la desactualización tecnológica manifiesta;

c) El abandono o la deserción de la empresa por parte de sus propietarios;

d) La reducción de personal por motivos económicos, el vaciamiento o el trasvasamiento de trabajadores, de maquinarias y de activos o la existencia de activos productivos ociosos;

e) La infracapitalización material sobrevenida, dificultades graves para cumplir compromisos, la suspensión de pagos o la falta grave de liquidez;

f) La inviabilidad sobrevenida, la decadencia empresarial, el desmantelamiento de unidades de producción o de ramas de actividad, y la incursión en persistentes distribuciones patrimoniales irregulares;

g) La precarización del trabajo, la ruptura de contratos de trabajo, reducciones, espaciado, suspensión o demoras en el pago de sueldos y salarios, el pago en vales o en bienes, materiales, productos o equipos de la empresa, deudas atrasadas con los trabajadores, falta de cumplimiento de los aportes a los sistemas de ahorro establecidos en beneficio de los mismos y, o a la seguridad social;

h) El incumplimiento reiterado e injustificado de obligaciones vencidas con el sistema financiero, con los organismos públicos de fomento, así como con los impuestos y contribuciones parafiscales;

i) La disolución de la empresa con causal de liquidación, el concurso de acreedores o la apertura de procedimiento concursal de la empresa; 
j) La privatización de empresas públicas, la expropiación de empresas privadas, su confiscación legalmente pronunciada, o su dación en pago;

\section{Artículo 6. Requisitos Financieros}

Para que sea procedente la recuperación de una empresa por parte de los trabajadores la misma debe tener posibilidades de funcionamiento sustentable desde el punto de vista económico y financiero, demostrable a través del cumplimiento de los siguientes requisitos:

a) Que sea factible su recuperación, manifestada en un Proyecto Financiero que contemple el plan de inversiones a realizar por parte de los trabajadores, las entidades financieras públicas o privadas y los propietarios que decían participar, si fuese el caso, y los acuerdos a que se llegare con los acreedores, los suministradores, subcontratistas, distribuidores, factoriados, concedentes y demás personas o entidades interesadas en la recuperación de la empresa;

b) Los aportes de los trabajadores sean representados en los activos de la empresa recuperada (inmuebles, muebles, derechos, obligaciones, títulos o acciones) mediante la conversión de la deuda laboral que tuviere el patrono con ellos, incluidos los intereses causados por la mora en su pago;

c) Los créditos de los propietarios de la empresa con los acreedores, el fisco, la Seguridad Social, las contribuciones parafiscales y cualquier otra entidad pública en ningún caso serán asumidos por los trabajadores, o traspasados a la forma jurídica que adopten los mismos, sino que seguirán al patrimonio personal de los propietarios, excusando la empresa en recuperación;

d) Los trabajadores podrán hacer aportes en la empresa en calidad de inversión, en los plazos y modalidades de acuerdo a la normativa la forma jurídica adoptada. también podrán hacerlo otras empresas sociales solidarias, los organismos de integración previstos en el Capítulo 5, las organizaciones internacionales de naturaleza semejante, los sindicatos y organizaciones de trabajadores, otras organizaciones sociales y comunitarias y entidades públicas nacionales o internacionales;

e) Las empresas cuya propiedad recupere el Estado por cualquier vía legal, podrán ser otorgadas a los trabajadores, a cuyos efectos serán consideradas como empresas recuperadas y estarán sujetas a la presente ley. 


\section{Artículo 7. Requisitos Técnicos}

La recuperación de una empresa en cierre por parte de los trabajadores sólo es posible técnicamente si:

a) Tiene comprobadas perspectivas de recuperación de su potencial productivo, de sus canales de suministro y, o de comercialización demostradas en un plan de producción, y, o así como si cuenta con maquinarias mínimamente adecuadas y capacidad instalada ociosa;

b) Es factible su actualización o innovación tecnológica y su reestructuración empresarial;

c) Contribuye efectivamente al desarrollo local auto-sustentable, preservando el medio ambiente;

d) Se dedica a sectores socioeconómicos definidos como prioritarios por el Estado, si fuese el caso; esté integrada a cadenas productivas de bienes y de servicios esenciales y primarios y, o se articule a redes asociativas económicas populares;

e) Se demuestre que los trabajadores tienen la capacidad técnica y administrativa necesaria, o que cuentan con la asesoría y la asistencia técnica del organismo de integración a que se refiere el Capítulo 5, o han llegado a acuerdos en tal sentido con otras personas o instituciones con experticia en la materia, preferentemente universitarias.

\section{Artículo 8. Requisitos Laborales}

La recuperación de la empresa por parte de los trabajadores depende de la comprobación de los siguientes extremos socio laborales:

a) La voluntad manifiesta de la mayoría de los trabajadores de querer participar;

b) Tener reales posibilidades de funcionamiento desde el punto de vista social y organizacional;

c) Pueda generar fuentes de trabajo decente según los Convenios y las Recomendaciones de la Organización Internacional del Trabajo;

d) Se respeten los derechos y las condiciones laborales de los trabajadores (incluida la higiene y la seguridad industrial) y se les otorgue protección de —al menos - los mismos sistemas de Seguridad Social establecidos en la Ley;

e) Se garantice que las remuneraciones de los trabajadores no sean inferiores al salario interprofesional prevaleciente en la zona;

f) Mantenga los valores, principios y características propias de la forma jurídica adoptada por la empresa, en especial, la vida asociativa de la misma. 
Las condiciones exigidas en los ordinales c) y e) de este artículo podrán ser temporalmente diferidas si fuese estrictamente necesario para la supervivencia de la empresa, siempre que cuente con el acuerdo de todos los trabajadores, y si con ello fuese posible su recuperación de acuerdo a los estudios técnicos correspondientes. En tal caso, se considerarán acreencias de los trabajadores, las que pueden ser imputadas como aportes de los mismos al capital social, una vez superadas las causas que la generaron.

\section{Artículo 9. Pérdida de la condición de empresa recuperada}

a) Son causales de pérdida de la condición de empresa recuperada, la disolución o la quiebra de la forma jurídica adoptada y el incumplimiento de los requisitos establecidos en el capítulo 2 de esta Ley. La conversión de forma jurídica, la fusión y la pertenecía a grupos empresariales por coordinación no son causa de pérdida de la condición de empresa recuperada, si se mantienen las características establecidas por el artículo 3;

b) La condición de empresa recuperada se extingue por decisión del Juez competente de acuerdo con el artículo 4 de esta Ley. Corresponde al organismo de integración al que se encuentre afiliada la empresa, certificar la pérdida de los requisitos de empresa recuperada, debidamente comprobada mediante la metodología del balance social. Si la empresa no estuviere afiliada a ningún organismo de integración, la certificación la hará el organismo de integración de la actividad más similar a ella;

c) La pérdida de la condición de empresa recuperada ocasiona la cesación, en un plazo perentorio, de los beneficios concedidos por el Estado de acuerdo con el artículo 12 de esta Ley, pasando los mismos al régimen ordinario establecido en la legislación nacional.

\section{CAPÍTULO TERCERO \\ Procedimiento de declaración de empresa recuperada}

\section{Artículo 10. Procedimiento amigable}

a) El proceso de recuperación de la empresa se inicia a solicitud expresa de los trabajadores en riesgo de perder sus trabajos, adoptada en una asamblea convocada por el sindicato o por los propios trabajadores interesados, acompañada de un anteproyecto de recuperación y de la comprobación de estar trami- 
tando la constitución de una forma jurídica cualquiera, presentada al Ministerio con competencia en el Trabajo.

b) La entrega de la solicitud ante el Ministerio otorga a los trabajadores la condición de terceros interesados, así como la guarda y custodia de los inmuebles de la empresa y de los bienes contenidos en ellos, para el cuidado, conservación y vigilancia de los mismos, con la finalidad de evitar pérdidas derivadas del abandono patronal;

c) En el momento de comprobarse haber llegado a un acuerdo los trabajadores con los propietarios y con la mayoría de los acreedores, -homologado por el Ministerio- se suspenderá cualquier concurso, liquidación o quiebra en curso y el mismo tendrá carácter de título para gozar de los beneficios establecidos en el Capítulo 4 de esta Ley para las empresas recuperadas;

d) Los trabajadores que no deseen participar en el proceso mantendrán incólumes sus créditos laborales, los que ejercitarán por la vía laboral ordinaria contra los propietarios.

\section{Artículo 11. Procedimiento judicial}

a) De no lograrse el avenimiento de las partes, o cuando no fuere posible la presencia de los propietarios o el acuerdo de la mayoría de los acreedores, los trabajadores que quieran recuperar la empresa presentarán al Juez competente los mismos requisitos exigidos en el literal a) del artículo 10;

b) El Juez, oído el Informe del Ministerio, del Síndico en caso de la quiebra, o de los liquidadores, decidirá en forma perentoria sobre la reiniciación o continuidad la actividad productiva bajo la responsabilidad de la forma jurídica adoptada por los trabajadores y la designará como depositaria judicial, mientras se deciden los recursos que pudieren interponerse por la vía ordinaria. El Juez podrá - además- designarla gestora delegada con facultades de simple administración e indicará los contratos en curso de ejecución que se mantendrán;

c) En caso de ser resueltos negativamente los recursos interpuestos, el Juez acordará a la forma jurídica empleada la condición de empresa recuperada con los efectos subsiguientes. Esta declaración produce —además - la disolución del contrato de trabajo de los trabajadores solicitantes y la transferencia de sus derechos laborales en calidad de aportes al capital social, tomando en cuenta el valor de realización de los activos; 
d) Si fuesen acordados los recursos interpuestos y negada la condición de empresa recuperada, los trabajadores participantes mantendrán sus créditos laborales indexados y se reconocerá como créditos adicionales al mayor valor producido a la empresa durante el período de la ocupación, los que tendrán igualmente el carácter de privilegio de primer grado;

e) En ambos procedimientos se concederá un plazo suficientemente amplio para que los trabajadores presenten un proyecto definitivo de recuperación de la empresa, que cumpla con los requisitos establecidos en el Capítulo 2 de esta Ley.

f) En cualquier estado del procedimiento tanto amigable como judicial, la autoridad que declare la expropiación de la empresa por causa de utilidad pública o social, la entregará en propiedad a los trabajadores interesados e indemnizará directamente al expropiado de acuerdo a la ley respectiva, otorgando a la forma jurídica adoptada por los trabajadores un crédito a largo plazo para su cancelación.

\section{CAPÍTULO CUARTO \\ Relaciones con el Estado}

\section{Artículo 12. Fomento del Estado}

Las entidades públicas administrativas y financieras nacionales, regionales y locales fomentarán y apoyarán a las empresas recuperadas por medio de las siguientes actividades:

a) El cofinanciamiento - en condiciones especiales - de los proyectos de recuperación de la empresa - tanto provisional como definitivo- que incluya partidas para inversiones en activos fijos, materias primas, maquinaria, propiedad industrial y equipamiento tecnológico;

b) Acceso a líneas de crédito y planes de financiamiento con intereses privilegiados para capital de giro y para la subsistencia de los trabajadores hasta la maduración del financiamiento otorgado, que les permita operar de acuerdo a las condiciones del mercado;

c) Se establecerá en la banca privada una cartera crediticia para las empresas recuperadas que permita cubrir déficits de financiamiento de parte de las entidades estatales, si fuese al caso;

d) El otorgamiento de avales a la empresa por parte de los Sistemas Nacionales de Garantías a los que se afilie la empresa recuperada; 
e) Derecho de preferencia —en primer grado- sobre la marca, las materias primas, insumos, maquinarias, herramientas, mercancías en proceso y terminadas, dinero efectivo y en cuentas bancarias, derechos, obligaciones, títulos valores y demás acreencias de que fuese titular la empresa en recuperación;

f) El pago único del total del monto de los seguros de desempleo que corresponda a los trabajadores de las empresas recuperadas desde el momento mismo del cierre empresarial, siempre que sea imputado al pago de sus aportes a la forma jurídica adoptada;

g) La cancelación a la nueva empresa de subsidios por el empleo creado y sostenido en el tiempo;

h) Otorgamiento de aportes no reembolsables para el diseño, evaluación y ejecución de proyectos y planes de inversión, para la contratación de gerentes experimentados, la adquisición de tecnologías e informática, asesorías especializadas y asistencia técnica, así como de actualización, especialización y profundización técnica, administrativa y cultural de los trabajadores;

i) Preferencias, en igualdad de condiciones, en licitaciones y contratos públicos, venta de activos, en las compras de los entes del Estado, en la prestación de servicios al mismo, y en las concesiones de obras y servicios, preferentemente de Servicios Públicos;

j) En la privatización de empresas del Estado, preferencia a sus trabajadores en la continuación de sus actividades;

k) Asesoramiento y orientación a los trabajadores en aspectos laborales, legales y de organización, preferentemente a través de universidades públicas.

\section{Artículo 13. Control}

a) El Estado ejercerá provisionalmente la fiscalización y el control del adecuado uso de los medios de apoyo otorgados por los entes públicos a las empresas recuperadas, a través del Ministerio con competencia en el Trabajo, o por medio de la Autoridad de Aplicación de la forma jurídica adoptada;

b) El ejercicio de las funciones de control a que se refiere el literal anterior será paulatinamente traspasado a los Organismos de Integración a que se refiere el artículo 15, para lo cual se establecerá un cronograma específico y se otorgarán los financiamientos necesarios para su puesta en marcha. 


\section{Artículo 14. Régimen impositivo}

a) Las empresas en proceso de recuperación recibirán un tratamiento preferencial en las actuaciones administrativas o judiciales en lo referente a derechos de publicaciones oficiales, escritura, sellos, timbres, tasas o aranceles judiciales, notariales o de registro;

b) Las empresas recuperadas gozarán de los mismos beneficios fiscales acordados en forma general por la ley respectiva a la forma jurídica adoptada;

c) La ley concederá un tratamiento fiscal favorable de carácter temporal y diferenciado, según el impacto que produzcan las empresas recuperadas en el mantenimiento y la generación de empleo, sujeto a certificación conforme a lo previsto en el artículo 9 de esta Ley.

\section{CAPÍTULO QUINTO \\ Integración y concentración}

\section{Artículo 15. Sistemas de integración operacional}

a) Las empresas recuperadas crearán y se afiliarán a los organismos de integración que consideren conveniente o adecuado a sus necesidades socioeconómicas, para el mejor cumplimiento de sus objetivos;

b) Los organismos e integración pueden ser específicos de las empresas recuperadas o ser los previstos por la normativa de la forma jurídica seleccionada;

c) Las empresas recuperadas podrán celebrar todo tipo de convenios, contratos, constituir cualquier tipo de asociaciones, sociedades o empresas, así como desarrollar distritos o cuencas empresariales con otras empresas recuperadas, solidarias, públicas o privadas de ámbito local, regional, nacional o internacional;

d) Las empresas recuperadas podrán constituir e integrar grupos y consorcios de empresas con centros unitarios de decisión, sin más limitaciones que las derivadas de su forma jurídica y de la legislación nacional;

e) Las empresas recuperadas podrán constituir y, o formar parte de empresas o de redes de empresas solidarias o de consorcios binacionales o regionales latinoamericanos de naturaleza social; 
f) Las empresas recuperadas podrán realizar actividades de complementación entre empresas relacionando los productos e insumos, compras conjuntas y vinculación con organismos tecnológicos del Estado y las universidades, con emprendimientos productivos y los movimientos de trabajadores desocupados;

g) Las empresas recuperadas extranjeras podrán operar en los demás países con su sola inscripción en el registro respectivo, según la forma jurídica que hubiesen adoptado.

\section{Artículo 16. Atribuciones de los organismos de integración}

a) Los organismos de integración a que se contrae el artículo anterior tendrán funciones de representación y defensa de los derechos e intereses de sus afiliadas, de su promoción y fomento, de registro, de asesoría, consultoría y asistencia técnica, de autocontrol de las operaciones de sus afiliadas, de certificación de la condición de empresa recuperada, de conciliación y arbitraje y las demás que acuerden sus estatutos;

b) Igualmente, podrán fomentar y establecer actividades productivas o de servicios conjuntos, conexos o complementarios que fuesen convenientes al cumplimiento de su objeto.

\section{Artículo 17. Integración Financiera}

a) Se establecerá un Fondo de Financiamiento de las empresas recuperadas, integrado inicialmente con aportes de las entidades Financieras del Estado y posteriores aportes de las empresas recuperadas, proporcionales a los aportes de aquellas, que será administrado con la participación de las entidades participantes en el mismo;

b) El Fondo cofinanciará los proyectos de recuperación de empresas, así como los sistemas de integración previstos en el artículo 15 de esta Ley. Igualmente, financiará las actividades de educación y de capacitación de los trabajadores;

c) Se proveerá en un plazo razonable a la creación y el fortalecimiento de organismos financieros propios de las empresas recuperadas para que asuman de forma autónoma las funciones de financiamiento a que se contrae el artículo 12 de la presente Ley, así como del Fondo Financiero previsto en el numeral anterior; 
d) De igual forma, se establecerán Sociedades de Garantía Recíproca propias de las empresas recuperadas, con la participación de otras empresas solidarias, si fuese el caso;

\section{Capítulo Sexto \\ Disposiciones Finales}

\section{Artículo 18. Disposiciones Transitorias}

a) Las empresas recuperadas que se encuentren funcionando al momento del inicio de la vigencia de la presente Ley, tendrán un plazo prudencial para sujetarse a las disposiciones de la misma;

b) Serán gratuitos los trámites judiciales y administrativos que se ocasionen a las empresas recuperadas por los actos que ejecuten para sujetarse a esta ley;

c) Los entes públicos a que se contrae la presente Ley deberán incluir en los presupuestos del año siguiente a su entrada en vigencia, las partidas necesarias para otorgar los financiamientos previstos en el literal a) del artículo 17 de esta Ley.

\section{Artículo 19. Disposición Derogatoria}

Se derogan todas las disposiciones legales que colidan con la presente Ley.

\section{Artículo 20. Vigencia}

La presente Ley entrará en vigencia en un lapso prudencial después de su publicación en el diario o gaceta oficial correspondiente.

Mérida, mayo de 2009. 